\title{
El Parámetro de los Compuestos en la adquisición del español como lengua extranjera considerando el tipo de lengua materna y el contexto de aprendizaje* .
}

\author{
The Compounding Parameter in the acquisition of \\ Spanish as a foreign language considering the influence of \\ the mother tongue and the learning context
}

\author{
Marta Trias, Ana María Villanueva \\ Departamento de Idiomas, Universidad Simón Bolivar. Venezuela \\ e-mail:mtrias@usb.ve,villanuevaam@usb.ve
}

Received: 29-Mar-2013 / Accepted: 27-May-2013

\section{Resumen}

El siguiente trabajo forma parte de un proyecto de investigación sobre el Parámetro de los Compuestos (Compounding Parameter) (Snyder 1995, 1996, 2001) en el marco teórico de la Gramática Universal. Según la tipología elaborada por Snyder (1995, 1996, 2001) las lenguas se caracterizan por la presencia o ausencia de compuestos [NN] productivos y de predicados complejos. Una prueba de juicio gramatical y una prueba de selección simple fueron empleadas con el propósito de observar las respuestas de los estudiantes de español como lengua extranjera, quienes fueron clasificados en dos grupos según el tipo de lengua materna, y el contexto de aprendizaje: instruccional (Estados Unidos) e instruccional + natural (Venezuela). Los resultados indican que el contexto natural + instruccional influye positivamente en la adquisición de algunas de las cuatro estructuras del parámetro estudiadas, asimismo indican una tendencia a la producción de estructuras $[\mathrm{NdeN}]$ y de [NN] con núcleo a la izquierda.

Palabras clave: Parámetro de los Compuestos, tipología de Snyder, compuestos nominales y predicados complejos.

\section{Abstract}

The present article is part of a research project on the Compounding Parameter (Snyder 1995, 1996, 2001) within the framework of Universal Grammar. According to the typology established by Snyder (1995, 1996, 2001), languages are categorized by the presence or absence of productive [NN] compounds and complex predicates. A grammatical judgment test and a multiple choice test were used to observe the answers of students of Spanish as a foreign language, grouped according to the type of mother tongue and learning context, instructional (US) or instructional + natural (Venezuela). Results indicate that the combination of natural + instructional learning context promotes the learning of some of the structures of the parameter studied in the article; there is also the tendency of the production of left headed [NdeN] and [NN].

Key words: Compounding Parameter, Snyder typology, noun compounds and complex predicates.

\section{Résumé}

Ce travail fait partie d'un projet de recherche sur le Paramètre des composés (Compounding Parameter) (Snyder 1995, 1996, 2001) dans le cadre théorique de la Grammaire Universelle. Selon la typologie élaborée par Snyder (1995, 1996, 2001), les langues se caractérisent par

* Este artículo reporta los resultados del proyecto de investigación titulado: Estudio del parámetro de los compuestos en una muestra de estudiantes de español como lengua extranjera, llevado a cabo en la Universidad Simon Bolivar, Caracas - Venezuela. Código del Proyecto: S1-IN-CSH-020-06. 
la présence ou l'absence de composés [NN] productifs et de prédicats complexes. Un test d'évaluation grammaticale et un test de sélection simple ont été appliqués afin d'observer les réponses des étudiants d'Espagnol comme langue étrangère. Ces derniers ont été classés en deux groupes, en fonction du type de langue maternelle et le contexte de formation : formation (États-Unis) et formation + origine (Venezuela). Les résultats indiquent que le contexte d'origine + contexte de formation a une influence positive dans l'acquisition de plusieurs des quatre structures du paramètre étudiées ; ces résultats montrent également une tendance à la production de structures [NdeN] et de [NN] dont le noyau est à gauche.

Mots clés: Paramètre des composés, typologie de Snyder, groupes nominaux et prédicats complexes.

\section{Resumo}

O seguinte trabalho forma parte de um projeto de pesquisa sobre o Parâmetro dos Compostos (Compounding Parameter) (Snyder 1995, 1996, 2001) no marco teórico da Gramática Universal. Segundo a tipologia elaborada por Snyder $(1995,1996,2001)$ as línguas se caracterizam pela presença ou ausência de compostos [NN] produtivos e de predicados complexos. Uma prova de julgamento gramatical e uma prova de seleção simples foram empregadas com o propósito de observar as respostas dos estudantes de espanhol como língua estrangeira, os quais foram classificados em dois grupos, segundo o tipo de língua materna, e o contexto de aprendizagem: instrucional (Estados Unidos) e instrucional + natural (Venezuela). Os resultados indicam que o contexto natural + instrucional influi positivamente na aquisição de algumas das quatro estruturas do parâmetro estudadas, da mesma forma, indicam uma tendência à produção de estruturas [NdeN] e de [NN] com núcleo à esquerda.

Palavras chave: Parâmetro dos Compostos, tipologia de Snyder, compostos nominais e predicados complexos.

\section{Introducción}

El presente estudio se suma a esfuerzos anteriores de las autoras Trías y Villanueva (2011) por explicar la adquisición de los nombres compuestos y los predicados complejos por parte de aprendientes de español como lengua extranjera (L2), a la luz de la teoría de la Gramática Universal (GU) y del Parámetro de los Compuestos (Compounding Parameter) (Snyder 1995, 1996, 2001). En el presente trabajo se estudiará la influencia de la lengua materna y el contexto de aprendizaje en la adquisición de las estructuras antes mencionadas.

Dentro del enfoque teórico de la GU, se ha estudiado el parámetro sintáctico llamado Parámetro de los Compuestos en la adquisición de lengua materna (L1) (Snyder 1995, 1996, 2001); y en la adquisición de L2 (Liceras y Díaz 2001; Slabakova 2002; Liceras, Salomaa-Robertson y Díaz 2002; Sugisaki y Snyder 2002; Isobe 2007) el cual tiene que ver con la riqueza que tienen ciertas lenguas en la formación de nombres compuestos del tipo Nombre + Nombre [NN]. Esta riqueza en la formación de compuestos nominales está relacionada con la presencia de predicados complejos tales como: el resultativo (Resultative), el verbo-partícula (Verb-
Particle), el poner locativo (Put Locative), el hacer causativo (Make Causative) y el doble objeto (Double Object) (Snyder 1995, 1996, 2001). Según Snyder (1995, 1996, 2001), Slabakova (2002), Liceras et al. (2001), en lenguas tales como el inglés se permite, no solamente la combinación de dos o más nombres en una sola cadena como una sola unidad, por ejemplo shoe lace (Slabakova 2002), sino que se permite la creación de nombres compuestos generados de forma espontánea por los nativos del inglés y comprensibles para los interlocutores, por ejemplo salamander jar (Slabakova 2002). Por el contrario, la lengua española como parte de la familia de las lenguas romance, no posee nombres compuestos del tipo [NN] con características productivas. Por lo tanto, el equivalente del compuesto hotel room en español requiere del uso de la preposición de para conectar los nombres, habitación de hotel, generando una estructura [NdeN].

Snyder (1995, 1996, 2001) sostiene que adicional a la composición nominal productiva, lenguas como el inglés exhiben construcciones de predicados complejos donde el verbo principal se combina con un predicado secundario y semánticamente parece 
un verbo simple (Isobe 2007; Sugisaki e Isobe 2000). Según Snyder (1996) los análisis que se han realizado en la lengua inglesa sobre los predicados complejos tratan el verbo principal y el predicado secundario de la construcción del predicado complejo como una unidad sintáctica y semántica. En las lenguas que no poseen compuestos [NN] productivos, como el español, esto no es posible y se hace necesario el uso de alguna preposición o la reformulación de la oración a través de una perífrasis. En caso contrario, el resultado es agramatical y de difícil interpretación. A continuación se ofrecen algunos ejemplos tomados de Snyder (1995, 1996, 2001):

1 John painted the house red. (Resultative) *Juan pintó la casa rojo. Juan pintó la casa de rojo/de color rojo. (Perífrasis con Frase Preposicional)

2 The natives waited out the crisis. (VerbParticle)

*Los nativos esperaron la crisis para afuera.

Los nativos esperaron hasta el final de la crisis. (Perífrasis con Frase Preposicional)

3 Alice sent Sue the letter. (Double Object)

*Alicia le envió Susana la carta. Alice sent the letter to Sue. (To-Dative) ${ }^{1}$ Alicia le envió la carta a Susana.

Sugisaki y Snyder (2002) retoman las ideas de Stowell de 1981 y 1982, quien sostiene que las lenguas que permiten la posibilidad de desplazar una preposición al final de una oración, lo que se conoce como Ramificación de la Preposición (Preposition Stranding) (ejemplo 4) forman parte de las mismas lenguas que permiten la construcción verbo partícula. En este sentido Sugisaki et al. (2002) explican que el Parámetro de los Compuestos es mucho más global de lo que había expuesto Snyder (1995, 1996, 2001) y que además de los predicados complejos citados anteriormente también se encuentra esta otra

1 Doble Objeto (Double Object) también es conocido en español como [Frase Nominal + Frase Nominal] (To-Dative) es conocido en español como [Frase Nominal + Frase Preposicional] estructura sintáctica como uno más de los correlatos de este parámetro.

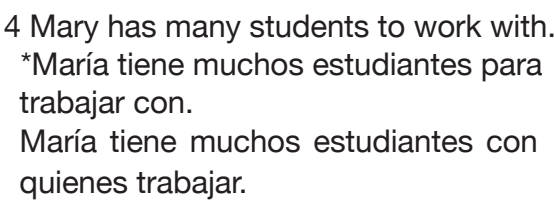

La ausencia de predicados complejos y de compuestos nominales productivos podría resultar particularmente difícil para aquellos aprendientes de español cuya lengua materna pertenece al grupo de lenguas que sí tienen estos rasgos de acuerdo a la tipología de Snyder (1995, 1996, 2001); o podrían resultar más fáciles para aquellos que comparten la tipología con el español. Por otro lado, es necesario tomar en cuenta, no sólo el tipo de lengua materna, sino la interacción con el tipo de input de la L2 que recibe el aprendiente (Bley-Vroman 1989). Según este autor, el aprendiente de L2 en un contexto instruccional de aprendizaje de la lengua está expuesto a un input limitado, a diferencia de los aprendientes en contexto natural cuyo grado de exposición al input es mayor. Por esta razón y a la luz de las consideraciones teóricas anteriores, nos hemos propuesto estudiar si el comportamiento de los sujetos de estudio con respecto a ciertas estructuras del Parámetro de los Compuestos se explica por el tipo de lengua nativa o por el contexto de aprendizaje en el que se encuentran (contexto instruccional y contexto instruccional + natural) o por la combinación de ambas variables. Este trabajo presenta una muestra de hablantes nativos de diferentes lenguas en dos contextos de aprendizaje opuestos que permite ampliar la investigación en el área. Dada la amplia variedad de correlatos asociados con el Parámetro de los Compuestos, en el presente estudio se han seleccionado solamente el resultativo, el verbopartícula, el doble objeto (con sus correspondientes versiones al español), los compuestos [NN] y la estructura [NdeN].

\section{Compuestos [NN] y predicados complejos en español}

De acuerdo con Snyder (1995, 1996, 2001) el español está clasificado como una de las lenguas 
en las que no existen combinaciones de [NN] de tipo productivo, en consecuencia tampoco existen predicados complejos de los tratados en el presente artículo: predicados de doble objeto, resultativos y con verbo partícula.

The Romance languages have long been noted to contrast with English and other Germanic languages in that they exclude resultative constructions of the type in 2d (John hammered the metal flat) ( $c f$ Green 1973, Kayne 1984, and especially Levin \& Rappaport 1988). Furthermore, the Romance languages systematically lack direct counterparts to the English verb-particle, make-causative, and double-object dative constructions. (Snyder 2001: 326)².

Los predicados de doble objeto, los resultativos y aquellos de verbo con partícula tienen sus equivalentes en formas perifrásticas en español. A continuación se detallarán cada uno de los componentes estudiados.

\section{Doble objeto}

En el grupo de las lenguas germánicas es posible encontrar construcciones que combinan objeto directo (OD) e indirecto (OI), como el siguiente ejemplo del inglés,

$$
\begin{aligned}
& 8 \text { He gave a flower to Mary. }(\mathrm{S}+\mathrm{V}+\mathrm{OD} \\
& +\mathrm{OI}) \\
& 9 \text { He gave Mary a flower. }(\mathrm{S}+\mathrm{V}+\mathrm{OI}+\mathrm{OD})
\end{aligned}
$$

En el ejemplo presentado en (9) el objeto indirecto (Mary) como benefactor de la acción del verbo give, se desplaza al lugar ocupado por el objeto directo en (8) (a flower), fenómeno que se conoce como construcción de doble objeto. En lenguas como el inglés, el holandés y el alemán (Demonte 1995) es posible usar tanto (8) como (9). En español, la presencia y el comportamiento del OD y el OI

2 Se ha notado desde hace tiempo que las lenguas romance contrastan con el inglés y con otras lenguas germanas en el hecho de que las lenguas romance excluyen las construcciones resultativas del tipo $2 \mathrm{~d}$ (John hammered the metal flat) (en Green 1973, Kayne 1984, y especialmente en Levin y Rappaport 1988). Además, las lenguas romance carecen sistemáticamente de construcciones directamente equivalentes a las que se conocen en inglés como el verbo con partícula, el make causativo, y el doble objeto dativo. (Snyder 2001, p. 326). Traducción libre de las autoras. en una misma oración son diferentes. La primera diferencia está basada en el hecho de que es necesario anteponer al complemento indirecto la preposición a / al / para, lo cual hace imposible las construcciones siguientes:

10 *El dio Mary una flor.

11 *El dio una flor Mary.

A pesar de que tanto el OD como el OI pueden intercambiar sus posiciones dentro de una misma oración, en español es necesario que el objeto indirecto esté igualmente precedido por a / al / para al ocupar el lugar del objeto directo.

La segunda diferencia está basada en la posibilidad de agregar el pronombre clítico le, inexistente en lenguas germánicas como las mencionadas anteriormente. En español, es posible duplicar el OI a través de la presencia del pronombre clítico y la forma explícita del objeto indirecto. En (12) y (13) la presencia del pronombre clítico le duplica la forma de dativo con preposición y produce una oración con doble objeto.

12 Él le dio a Mary una flor.

13 Él le dio una flor a Mary.

Algunos autores como Demonte (1995), afirman que sí existen construcciones de doble objeto en español, las cuales corresponden a aquellas en las que se usan formas del pronombre clítico le / les. A pesar de estas aseveraciones, el presente trabajo solo tomará como referencia la afirmación tradicional de que no existe una forma exacta de la construcción de doble objeto tal como la que aparece en (9).

\section{Resultativos}

Un resultativo es un tipo de predicado secundario que añade información acerca del efecto obtenido como consecuencia de la acción de un verbo transitivo. En el caso del predicado resultativo en inglés, el verbo principal se combina con un adjetivo para completar el significado del verbo y presentar 
el resultado de la acción sobre el objeto directo. En español, los predicados resultativos se pueden expresar a través de una oración subordinada encabezada por una preposición. En español es posible agregar un adjetivo al verbo para hacer una estructura como en John painted the house red, pero la oración resulta ambigua, como el caso de (14). Es necesario el uso de la preposición de para aclarar el sentido como en el caso (15).

14 Eduardo pintó la casa roja.

15 Eduardo pinto la casa de rojo.

\section{Verbo partícula}

El español no tiene verbos con partículas, y depende sólo del significado del verbo. Según la tipología de los verbos de movimiento elaborada por Talmy $(1985,1991,2000)$ se puede distinguir entre aquellas lenguas que expresan dirección del movimiento path of movement llamadas también verb-framed languages (V-language) como el español y aquellas que expresan el movimiento del verbo a través de una partícula o preposición asociada al verbo conocidas como satellite-framed languages (S-language) o manner languages como el inglés.

16 Mary lifted the box up. (Snyder 2005)

17 María levantó la caja. ( ${ }^{\star}$ arriba).

En lenguas como el inglés y el alemán, el verbo expresa el acto de movimiento junto con información sobre la manera, la forma en la que el movimiento se desarrolla. En el caso del español o el francés, el movimiento se expresa en el verbo y la manera en que se desarrolla puede ser agregada opcionalmente.

18 La botella entró a la cueva. (flotando)

(Talmy 1985)

19 The bottle moved-in to the cave. (floating)

20 The bottle floated into the cave.

La tipología de Talmy $(1985,2000)$ coincide con la tipología desarrollada por Snyder (1995, 1996, 2001). Según Snyder (2005) y Snyder et al. (2005), en el caso de las lenguas satellite-frame o $[+\mathrm{TPC}]^{3}$ las construcciones de verbo-partícula, así como los resultativos transitivos y las creaciones noveles de compuestos nominales son posibles; pero en las lenguas del tipo verb-frame o [-TPC] estos predicados no son posibles como tampoco es posible la creación de compuestos nominales de forma espontánea.

\section{Compuestos $[N+N]$}

El español no posee compuestos [NN] productivos, es decir, composiciones de Nombre + Nombre que puedan acuñarse según las necesidades de comunicación en un momento dado, y cuyo significado sea interpretado por el receptor, tal como sucede en lenguas como el inglés. Las combinaciones [NN] que presenta el español son altamente lexicalizadas, están consolidadas en la lengua con significado "propio, unitario y constante con un referente único.” (Varela 2005 p. 73). Los compuestos [NN] como bocacalle, hombre lobo, forman parte del léxico establecido y no pueden ser improvisados en el uso por parte de los hablantes, sin correr el riesgo de ser incomprendidos. En lugar de compuestos [NN], el español favorece la construcción de [NdeN] (fuerza de trabajo, árbol de manzana, mata de mango). Otros compuestos, como los del tipo [VN], (sacapuntas, guardacostas), o del tipo [N+Adj] (pelirrojo) son mucho más productivos en español.

Es importante señalar también que los compuestos [NN] en español tienen el núcleo a la izquierda y el modificador a la derecha, contrario al inglés, y las marcas morfológicas de género y número no siempre afectan al compuesto en su totalidad. La tendencia parece ser la de adoptar las marcas de género y número presentes en el núcleo, por ejemplo horas pico, faldas pantalón.

\section{Clasificación de las lenguas según Snyder}

Snyder $(1995,1996,2001)$ elaboró una tipología considerando como base la productividad de los compuestos [NN] y de los predicados complejos del

3 [+/- TPC] tipología del parámetro de los compuestos (Snyder 2005; Snyder y Lillo Martin 2005). 
tipo resultativo, verbo-partícula, doble objeto, hacer causativo. Esta tipología reúne a aquellas lenguas que poseen [NN] productivos y predicados verbales complejos dentro de una misma categoría, y en otra categoría a aquellas lenguas que no los poseen de forma productiva. Según esta tipología, si una lengua posee predicados complejos del tipo antes mencionados también poseerá compuestos [NN]. Snyder $(1995,1996,2001)$ elaboró su tipología tomando como base dos vertientes: a) la observación interlingüística, y b) el análisis de la muestra de 12 niños hablantes de inglés de CHILDES.

En la Tabla $N^{\circ} 1$ se muestra la clasificación ofrecida por Snyder $(1996,2001)$ y Slabakova (2002).

Tabla No 1 Clasificación de las lenguas según Snyder (1996, 2001) y Slabakova (2002)

\begin{tabular}{|c|c|c|}
\hline & RESULTATIVOS & $\begin{array}{l}\text { COMPUESTOS NN } \\
\text { PRODUCTIVOS }\end{array}$ \\
\hline $\begin{array}{l}\text { INGLES } \\
\text { HOLANDES } \\
\text { ALEMAN } \\
\text { HUNGARO } \\
\text { FINES/FINALANDES } \\
\text { KHMER } \\
\text { SUECO * } \\
\text { NORUEGO * }\end{array}$ & $\begin{array}{l}\mathrm{SI} \\
\mathrm{SI} \\
\mathrm{SI} \\
\mathrm{SI} \\
\mathrm{SI} \\
\mathrm{SI}\end{array}$ & $\begin{array}{l}\text { SI } \\
\text { SI } \\
\text { SI } \\
\text { SI } \\
\text { SI } \\
\text { SI }\end{array}$ \\
\hline $\begin{array}{l}\text { COREANO } \\
\text { JAPONES } \\
\text { CHINO MANDARIN }\end{array}$ & $\begin{array}{l}\text { NO (Snyder 1996) } \\
\text { SI (Snyder 2001) }\end{array}$ & $\begin{array}{l}\text { NO (Snyder 1996) } \\
\text { SI (Snyder 2001) }\end{array}$ \\
\hline $\begin{array}{l}\text { ARABE PALESTINO } \\
\text { FRANCES } \\
\text { ESPANNOL } \\
\text { HEBREO MODERNO } \\
\text { RUSO } \\
\text { SERBO-CROATA } \\
\text { PORTUGUES * } \\
\text { ITALIANO * }\end{array}$ & $\begin{array}{l}\text { NO } \\
\text { NO } \\
\text { NO } \\
\text { NO } \\
\text { NO } \\
\text { NO }\end{array}$ & $\begin{array}{l}\text { NO } \\
\text { NO } \\
\text { NO } \\
\text { NO } \\
\text { NO } \\
\text { NO }\end{array}$ \\
\hline
\end{tabular}

*Lenguas presentes en nuestro estudio que no aparecen reportadas por Snyder. Según lo consultado con los hablantes nativos del sueco, los compuestos nominales son productivos. Según Comrie (1990), el danés, el noruego y el sueco pertenecen a la familia de lenguas germanas, por esta razón están incluidas en el grupo de las lenguas productivas. El italiano y el portugués forman parte de las lenguas romance, las cuales no son productivas. Las tipologías de Snyder $(1995,1996,2001)$ y la de Talmy (1985) y Talmy (1991) y (2000) (en Snyder, W. 2005; Snyder et al. 2005) coinciden considerablemente (Snyder 2005).

Papel del input y del contexto de aprendizaje en la adquisición de una L2

El papel del input que recibe el niño o el aprendiente de una L2 ha sido ampliamente estudiado y su importancia ha variado según el enfoque teórico (Gass 1997, 2003; Gass et al. 2008). Dentro del marco teórico de la GU la importancia atribuida al input es menor que en otros enfoques ya que éste no suministra la información necesaria para formar abstracciones sobre la lengua - input empobrecido-, las cuales le permiten al niño adquirir conocimiento sobre la gramaticalidad o agramaticalidad en su lengua materna (White 1989, 2000). Para la GU, la lengua consiste en un conjunto de principios abstractos comunes a todas las lenguas y de parámetros - variabilidad entre las lenguas - que 
forman las gramáticas centrales de las lenguas (Gass 1997, 2003). Siguiendo las nuevas propuestas del Programa Minimista, las diferencias paramétricas entre las gramáticas están asociadas a propiedades léxicas llamadas categorías funcionales y éstas tienen rasgos formales asociados (género, número, caso) que forman parte del inventario de la GU. Estas categorías funcionales y rasgos formales, así como la fuerza de estos rasgos pueden variar de una lengua a otra (White 2003). La adquisición se produce sobre la base de la interacción del input con los principios y parámetros/categorías funcionales y rasgos formales de la GU (White 2000; 2003).

La exposición al input en la adquisición de la L1 ocurre básicamente sobre la base de la evidencia positiva, la cual se refiere al conjunto de oraciones formadas gramaticalmente al que el niño es expuesto (Gass 2003). En contraste, White (1989) sostiene que la evidencia negativa consiste en información sobre la no-gramaticalidad proveniente de la enseñanza de la gramática y de la corrección de errores. La adquisición de la L2 ocurre mayormente sobre la base de la evidencia negativa (Gass 1997, 2003; White 1989; Bley-Vroman 1989). Según Gass (2003), la evidencia negativa puede darse antes de producirse el error (pre-emptive) que viene a ser la que ocurre en situación formal de clase, y la evidencia negativa (reactive) que es posterior a la emisión de un error, y que puede ser explícita o implícita. En la adquisición de una lengua extranjera, el aprendiente se enfrenta a la tarea de darle sentido al input de la L2 que recibe - a la evidencia positiva pero básicamente negativa - lo que le permitirá comprender y producir estructuras de la L2 (White1989). Según White (1989; 2003) la adquisición de la L2, a diferencia de la L1, ocurre en un ambiente instruccional (situación formal de clases) y/o natural (inmersión) con mayor evidencia negativa que positiva y donde la variabilidad del input dependerá del ambiente de aprendizaje y del método de enseñanza (White1989). Para Bley-Vroman (1989), según profesores y estudiantes de L2 la evidencia negativa es útil en muchas ocasiones, ya que permite revertir ciertas hipótesis y ajustar las estructuras en la interlengua. Según este autor, un aprendiente de L2 en un contexto instruccional está expuesto a un input limitado ya que la lengua meta no se habla en el país y el número de horas de clase es limitado, a diferencia de los aprendientes en contexto natural cuyo grado de exposición al input es mayor, aunque esto último no siempre garantiza el éxito. Algunas de las razones que pueden explicar el logro en la adquisición de la L2 podrían tener que ver con diversos factores como la edad, el nivel educativo, la actitud hacia lengua, entre otros.

\section{Antecedentes del estudio}

Slabakova (2002) estudió el Parámetro de los Compuestos con grupos de estudiantes de español como lengua extranjera, clasificados de la siguiente manera: tres grupos de hablantes nativos del inglés distribuidos en tres niveles de adquisición de la lengua, otro grupo de hablantes nativos del francés principiantes, y un último grupo de control. La autora usó una prueba de juicio gramatical y una prueba de selección para evaluar el desempeño de los aprendientes en los distintos correlatos del parámetro (resultativos, doble objeto, verbo-partícula y [NN]). En el estudio, la autora se propuso probar dos hipótesis, la hipótesis de transferencia y la hipótesis del principio de subconjunto ${ }^{4}$ para la comparación entre los dos grupos de principiantes. Según la primera hipótesis, los francófonos deberán ofrecer mejores resultados en ambas pruebas dado que el español y el francés no poseen las estructuras estudiadas. La segunda hipótesis sostiene que si el principio de subconjunto opera efectivamente, tanto los anglófonos como los francófonos principiantes rechazarán desde el inicio las estructuras agramaticales para el español al ser

4 White (1989, p. 148) sostiene que el principio de subconjunto es un principio de aprendizaje distinto de la GU pero que interactúa con ella. Este principio debe ayudar al aprendiz de una L1 a hacer la selección correcta entre los diferentes valores de un parámetro al estar expuesto a la lengua meta. Sin embargo, según esta autora, este principio no opera efectivamente en la adquisición de la L2, ya que los aprendientes no empiezan con el valor paramétrico más restrictivo compatible con los datos de la L2 sino que adoptan consecuencias más generales basadas en la L1. (White, 1989, p. 164). 
expuestos al input de la L2 (español). Los resultados del estudio para los principiantes comprueban la primera hipótesis dado que los francófonos exhibieron un mejor desempeño en ambas pruebas a pesar de tener ambos grupos el mismo nivel de suficiencia en el español. Según Slabakova (2002), para que la segunda hipótesis pudiera darse, no bastaría solamente con la exposición al input (evidencia positiva) sino que sería necesaria la evidencia negativa también.

En cuanto a los grupos anglófonos del nivel intermedio y avanzado, el interés de la autora es ver si estos aprendientes fijan el valor del parámetro para el español en los cuatro correlatos. Estos grupos ofrecen un mejor desempeño que los anglófonos principiantes sobre todo en las estructuras [NN] y de doble objeto, pero no así para las estructuras de verbo partícula y sobre todo de resultativo. Según la autora, los aprendientes pudieron haber fosilizado esta forma agramatical del resultativo en el español. Los resultados positivos ofrecidos por estos aprendientes en el [NN] y el doble objeto podrían ser explicados por la exposición a la evidencia positiva y negativa, ya que al recibir instrucción formal los aprendientes fueron informados sobre la agramaticalidad de los $[\mathrm{NN}]$ y el doble objeto en el español, aunque ese no sería el caso para el verbo partícula y el resultativo. Los resultados indican que los aprendientes parecen fijar el valor del español para una parte de los correlatos del parámetro, pero no para la otra; por tanto, la hipótesis de que al adquirir un correlato se adquirirán los demás correlatos del parámetro como un cluster no puede ser confirmada con los resultados de ese estudio para la totalidad de los sujetos (Slabakova 2002).

Liceras et al. (2001) y Liceras et al. (2002) realizaron estudios sobre los compuestos nominales del tipo [NN] lo que constituye una parte de los correlatos (cluster) del Parámetro de los Compuestos.

Liceras et al. (2001) observaron el desempeño de aprendientes del español con 2 grupos de estudio constituidos por hablantes de lenguas indoeuropeas por una parte, $\mathrm{y}$ de hablantes de lenguas no indo-europeas por la otra, en el uso de los compuestos nominales. Los sujetos se encontraban en dos ambientes diferentes de aprendizaje: ambiente instruccional (Canadá) y ambiente instruccional + natural (España). Liceras et al. (2001) utilizaron dos pruebas de evocación con dibujos cuyo objetivo era evaluar: a) el conocimiento de los sujetos con respecto al bajo grado de productividad de los [NN] en español, opción marcada del parámetro y, b) el conocimiento de la direccionalidad (izquierda-derecha) y de la marca del género (femenino-masculino) [+rasgo de marcador palabra] de los [NN]. Los resultados mostraron que la producción de [NN] es una estrategia productiva en todos los grupos, menos en el grupo de principiantes de España, por lo que la producción de [NN] no constituye la opción marcada del parámetro - lo cual es contrario a lo que había establecido Snyder (1995) - y que la direccionalidad constituye un problema para todos los principiantes, sobre todo para los de Canadá. Con respecto a la selección de [NN] o de [NdeN], se observó que la selección de [NN] aumenta a medida que se incrementa el nivel de suficiencia de la lengua y que los sujetos en Canadá favorecen la producción de [NN] mientras que los sujetos en España prefieren [NdeN] con núcleo hacia la derecha especialmente los sujetos de lenguas no indo-europeas.

Liceras et al. (2002) realizaron un estudio sobre los [NN] y el rasgo marcador palabra con aprendientes de español nativos del inglés, del francés y del finés. Los instrumentos empleados fueron una tarea de descripción de imágenes y una de paráfrasis. Los resultados mostraron que la producción de [NN] con dirección hacia la izquierda o hacia la derecha es una estrategia muy productiva, del mismo modo que reporta Liceras et al. (2001) en su estudio. Sobre la base de ambos estudios (Liceras et al. 2001; Liceras et al. 2002) se deduce que la producción de [NN] no constituye la opción marcada del Parámetro de los Compuestos tal como lo había expresado Snyder (1995). Los grupos de francés y de inglés ofrecen un desempeño uniforme en la selección de estructuras 
[NN] o [NdeN] con núcleo a la izquierda. Sin embargo, el desempeño del grupo de finés es muy diferente al de los otros dos grupos, ya que seleccionan un mayor número de [NN] o de [NdeN] con núcleo a la derecha. Por último, Liceras et al. (2002) mencionan que los resultados ofrecidos en su estudio se deben, por una parte, a una carencia de input adecuado debido al escaso contacto de los sujetos con compuestos [NN], ya que éstos no son productivos en el español y tampoco son tratados en los libros de texto; y por otra parte, a la imposibilidad de estos sujetos de acceder al marcador palabra (marca femenino-masculino) de los sustantivos del español.

\section{Metodología}

Las lenguas nativas de los sujetos del estudio fueron agrupadas según la tipología de Snyder (1995, 1996, 2001) según la cual existen lenguas ricas en [NN] productivos y predicados complejos, a las que llamaremos [L1+], y lenguas pobres en [NN] productivos y predicados complejos, a las que llamaremos [L1-].

La tabla $N^{\circ} 2$ resume el diseño del estudio para el cual se toma en cuenta el tipo de lengua materna [L1+] o [L1-], el tipo de estructura morfosintáctica y el contexto de aprendizaje de la L2.

Tabla 2. Diseño del estudio con las combinaciones del tipo de lengua materna [L1+] o [L1-], el tipo de estructura y el contexto de aprendizaje

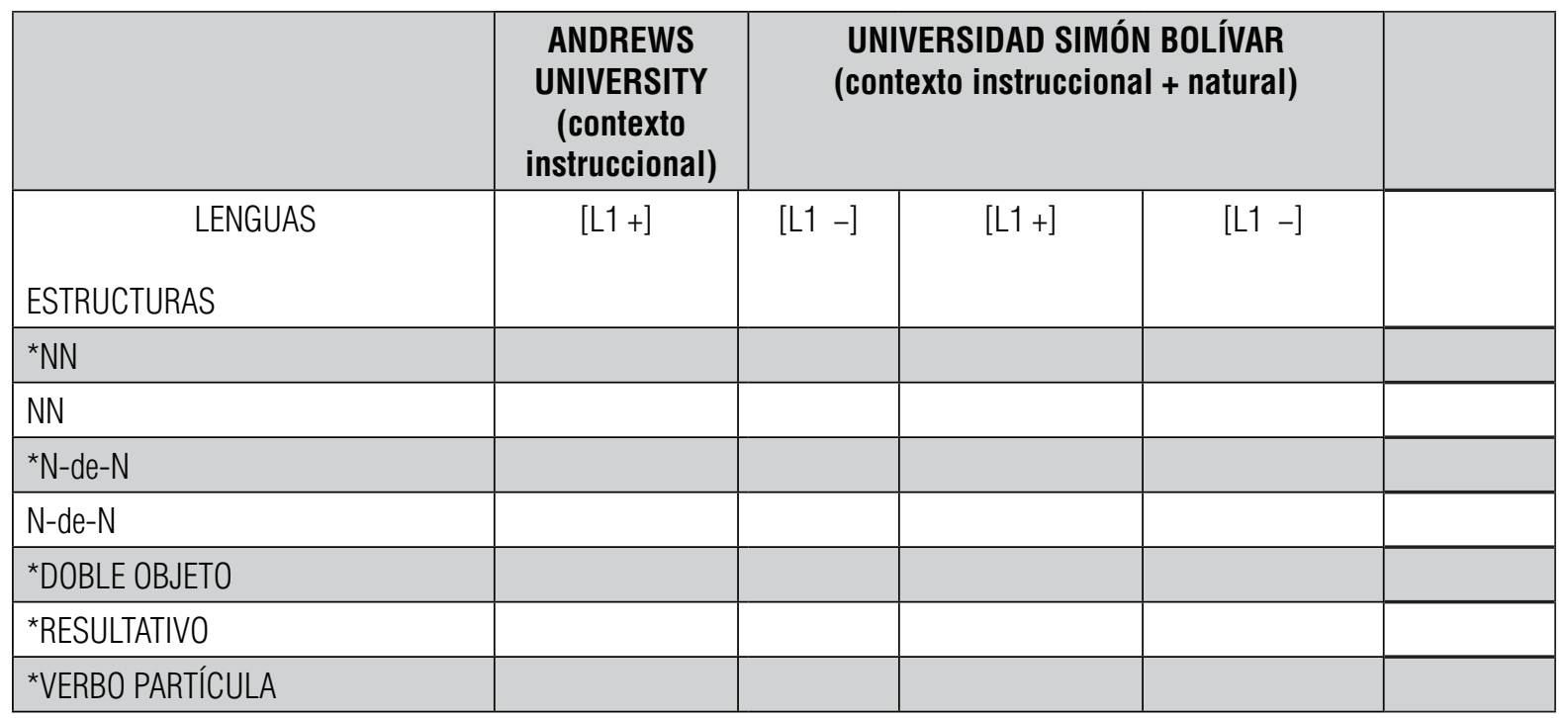

Con el fin de estudiar si el tipo de lengua materna o el contexto de aprendizaje (o la combinación de ambas variables) inciden en la adquisición de ciertas estructuras del Parámetro de los Compuestos, nos hemos formulado los siguientes objetivos:

Observar el desempeño global de los hablantes de [L1+] y [L1-] en la prueba de juicio gramatical y la prueba de selección simple según la situación de adquisición-aprendizaje de español como L2.

Observar el desempeño de los hablantes de [L1+] y [L1-] en 4 de los componentes del parámetro empleados en la prueba de juicio gramatical según la situación de aprendizaje.

Observar el desempeño de los hablantes de $[\mathrm{L} 1+]$ y [L1-] en las estructuras [NN] y [NdeN] en la prueba de selección simple según la situación de aprendizaje.

Observar el desempeño de los hablantes de [L1+] y [L1-] en las estructuras [NN] y [NdeN] (con versión gramatical y agramatical) en la prueba de selección simple según la situación de aprendizaje. 


\section{Sujetos}

Los sujetos que participaron en el estudio fueron $N=105$ : 66 sujetos que estudiaban español en Andrews University en los Estados Unidos (EEUU), 39 sujetos que estudiaban español en la Universidad Simón Bolívar en Venezuela (VZLA) y 20 hablantes nativos de español que participaron como grupo de control, quienes eran estudiantes regulares de la misma universidad venezolana. Los participantes de EEUU eran en su mayoría hablantes nativos de inglés en cambio los sujetos de VZLA eran hablantes nativos de diferentes lenguas (sueco, finés, francés, inglés, italiano, alemán, noruego, húngaro y portugués). Es importante señalar que los sujetos participantes de EEUU se encontraban estudiando español como lengua extranjera en un medio angloparlante y en un contexto instruccional, mientras que los sujetos de VZLA se encontraban estudiando español en un medio hispanohablante, es decir contexto instruccional y natural. La Tabla No $^{\circ}$ resume el número de sujetos participantes en el estudio y la $\mathrm{N}^{\circ} 4$ muestra el número de sujetos según su lengua materna.

Tabla 3. Sujetos del estudio

\begin{tabular}{|c|l|l|}
\hline & \multicolumn{1}{|c|}{ Grupos experimentales } & \multicolumn{1}{c|}{ Grupo de control } \\
\hline Andrews University & 66 sujetos & \\
\hline Universidad Simón Bolívar & 39 sujetos & 20 sujetos \\
\hline
\end{tabular}

Tabla 4. Sujetos según su lengua materna

\begin{tabular}{|c|c|}
\hline Lengua materna (L1) & N \\
\hline inglés & 61 \\
\hline francés & 14 \\
\hline portugués & 3 \\
\hline italiano & 1 \\
\hline noruego & 2 \\
\hline alemán & 10 \\
\hline húngaro & 1 \\
\hline finés & 1 \\
\hline sueco & 12 \\
\hline Total & 105 \\
\hline
\end{tabular}

Todas las lenguas que aparecen en la Tabla $\mathrm{N}^{\circ}$ 4 fueron catalogadas según la presencia o ausencia de las estructuras de predicados complejos y de la productividad de nombres compuestos del tipo [NN] tomando como base la clasificación de Snyder (1995, 1996, 2001) y Slabakova (2002). Snyder (1995, 1996, 2001) solo clasifica las lenguas según la productividad de los compuestos [NN] pero no hace mención a la direccionalidad de los compuestos en cada una de estas lenguas.

En la Tabla $N^{\circ} 5$, se presentan las lenguas nativas de los sujetos de estudio clasificadas según riqueza/pobreza en la producción de [NN] y de los predicados complejos para cada uno de los grupos experimentales. 
Tabla 5. Lenguas nativas de los sujetos de estudio clasificadas según riqueza/pobreza en la producción de [NN]

\begin{tabular}{|l|c|c|c|}
\hline & {$[\mathrm{L} 1+]$} & {$[\mathrm{L} 1-]$} & Total \\
\hline Andrews University (EEUU) & 60 & 6 & 66 \\
\hline Universidad Simón Bolívar (VZLA) & 27 & 12 & 39 \\
\hline Total & 87 & 18 & 105 \\
\hline USB grupo de control & 0 & 20 & 20 \\
\hline
\end{tabular}

\section{Instrumentos}

Se utilizaron dos pruebas para la recolección de los datos, una prueba de juicio gramatical (PJG) y una prueba de selección simple (PSS), adaptadas de Slabakova (2002). La PJG consiste en 56 oraciones

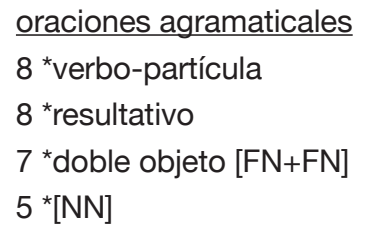

Por otra parte, la PSS consta de 17 ejercicios de selección simple en los cuales un enunciado debe ser completado usando una de las tres opciones ofrecidas. Estas opciones presentan tres de las siguientes estructuras:

estructura [NN] con núcleo a la izquierda (falda pantalón)

estructura *[NN] con núcleo a la derecha ("espejo laberinto)

estructura [NdeN] con núcleo a la izquierda (torta de manzana)

estructura *[NdeN] con núcleo a la derecha ("poeta de prodigio)

Ejemplo:

Es importante salir antes de las 6:00 de la tarde para evitarnos el tráfico de la

pico hora.

hora pico.

hora de pico. en español presentadas en orden aleatorio, de las cuales 28 son gramaticales y 28 agramaticales. Las oraciones estaban distribuidas de la siguiente manera:

\section{oraciones gramaticales}

8 verbo-partícula perifrástico

8 resultativo perifrástico

7 a-dativo (to Dative) [FN+FP]

5 [NdeN]

En algunos casos, la opción correcta es un [NN] con núcleo a la izquierda, como en el ejemplo antes mencionado, y en otros casos, la opción correcta es un [NdeN] con núcleo a la izquierda. Cabe destacar que en algunos casos se ofrece un [NdeN] con núcleo a la izquierda que no constituye la opción correcta para completar el enunciado de la prueba, aunque esa opción podría ser posible en otro contexto, por ejemplo: *química de laboratorio [NdeN] opción incorrecta para el enunciado porque es un [NdeN] con núcleo a la derecha. La opción correcta es entonces laboratorio de química [NdeN] con núcleo a la izquierda porque el enunciado dice trabajar en el.... Otros ítems con similares características son los números 11, 12, 13 y 14.

La PSS se utilizó con el fin de ofrecer a los sujetos una mayor variedad de estructuras nominales: a) [NN] con núcleo a la izquierda y a la derecha, y b) [NdeN] con núcleo a la izquierda y a la derecha.

Antes de aplicar las pruebas mencionadas, se agregó un cuestionario que tenía como objeto 
conocer la lengua materna del sujeto, la lengua de los padres, la lengua hablada en casa y el conocimiento de otras lenguas. Después de leer los cuestionarios fue necesario eliminar algunos sujetos del grupo de Estados Unidos que tenían conocimiento del español, bien sea porque sus padres eran hablantes nativos de la lengua o porque era la lengua hablada en casa.

Debido a que las pruebas utilizadas sufrieron alguna transformación con respecto a la original de Slabakova (2002), las mismas fueron validadas con sujetos estudiantes de español como segunda lengua que no forman parte de la muestra.

\section{Resultados}

A continuación presentamos las tablas $\mathrm{N}^{\circ} 6 \mathrm{y} \mathrm{N}^{\circ}$ 7 que revelan el desempeño global de los hablantes de [L1+] y [L1-] en las pruebas PJG y PSS según la situación de adquisición-aprendizaje de español como L2.

Tabla 6. Desempeño global de los grupos experimentales en la PJG

\begin{tabular}{|c|c|c|c|c|}
\hline Lugar de estudio & Lengua & Media & $\mathbf{~ N}$ & Desv. típ. \\
\hline EEUU & L1- &, 7470 & 6 &, 11921 \\
\hline & L1+ &, 5798 & 60 &, 11059 \\
\hline & Total &, 5950 & 66 &, 12059 \\
\hline & L1- &, 8244 & 12 &, 11619 \\
\hline VZLA & L1+ &, 7222 & 27 &, 09608 \\
\hline & Total &, 7537 & 39 &, 11183 \\
\hline & L1- &, 7986 & 18 &, 11968 \\
\hline Total & L1+ &, 6240 & 87 &, 12480 \\
\hline & Total &, 6539 & 105 &, 13998 \\
\hline
\end{tabular}

Tabla No $^{\circ}$ Desempeño global de los grupos experimentales en la PSS

\begin{tabular}{|c|c|c|c|c|}
\hline Lugar de estudio & Lengua & Media & N & Desv. típ. \\
\hline \multirow{2}{*}{ EEUU } & L1- &, 7745 & 6 &, 10132 \\
\cline { 2 - 5 } & L1+ &, 5918 & 60 &, 13639 \\
\cline { 2 - 5 } & Total &, 6084 & 66 &, 14309 \\
\hline \multirow{2}{*}{ VZLA } & L1- &, 7990 & 12 &, 14068 \\
\cline { 2 - 5 } & L1+ &, 7119 & 27 &, 15702 \\
\cline { 2 - 5 } & Total &, 7387 & 39 &, 15575 \\
\hline Total & L1- &, 7908 & 18 &, 12636 \\
\hline & L1+ &, 6291 & 87 &, 15277 \\
\hline & Total &, 6568 & 105 &, 16019 \\
\hline
\end{tabular}

En estas tablas se puede apreciar que los sujetos de [L1-] VZLA obtienen los mejores resultados (PJG ,8244 y PSS ,7990), mientras que los sujetos de [L1+] EEUU obtienen los resultados más bajos (PJG ,5798 y PSS ,5918), lo que constituye el efecto esperado.
Con respecto a la lengua materna, al comparar los grupos de [L1+] y [L1-] dentro de una misma situación de aprendizaje, se observa que los dos grupos de [L1-] obtienen mejores resultados que los de $[\mathrm{L} 1+]$ en ambos contextos, lo cual parece 
indicar que el tipo de lengua materna influye en los resultados.

En lo que concierne al contexto de aprendizaje, el rendimiento es mejor en el contexto VZLA que en el contexto EEUU, tanto para [L1+] como para [L1-]. Ambos grupos de VZLA, tanto el de [L1-] como el de $[\mathrm{L} 1+]$, obtienen mejores resultados que sus pares en
EEUU; es decir, que la situación de contexto natural + instruccional pareciera facilitar el proceso de aprendizaje.

El gráfico $N^{\circ} 1$ muestra el desempeño de los hablantes de [L1+] y [L1-] en el uso de los componentes del parámetro empleados en la PJG según la situación de aprendizaje, segundo objetivo de nuestro estudio.

Gráfico 1. Comparación contexto-tipo de lengua en las cuatro estructuras del PJG

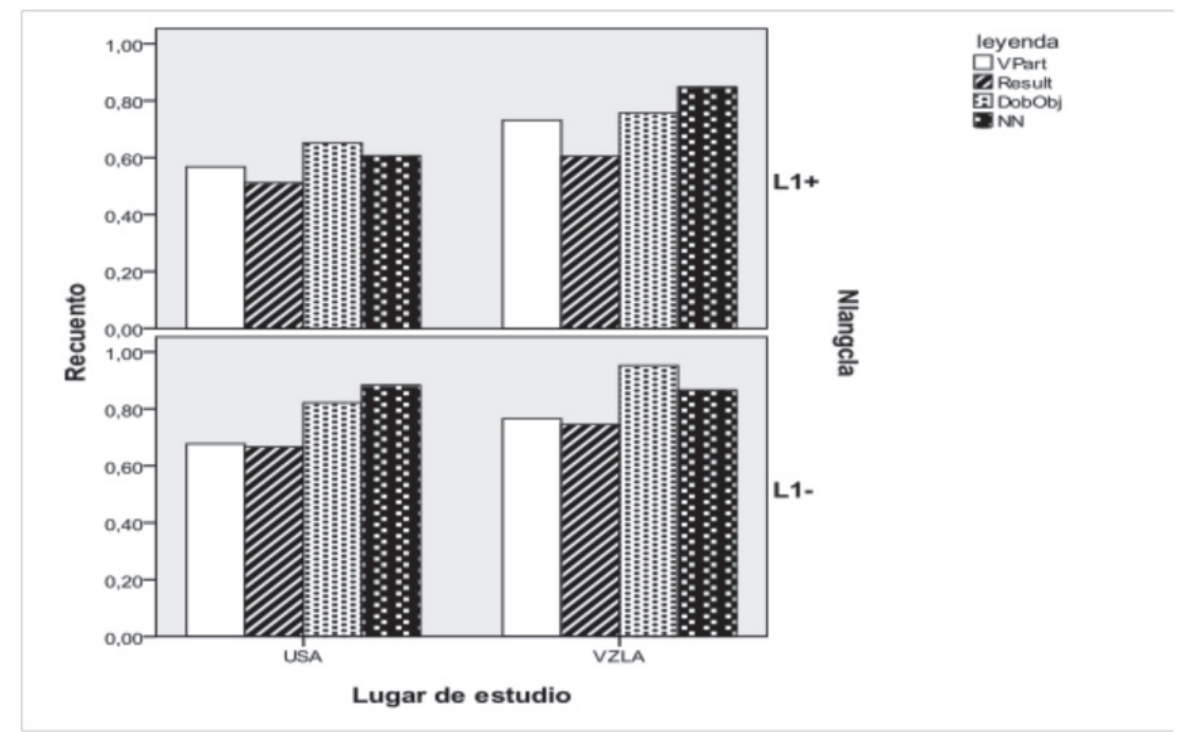

En cuanto al tipo de lengua, se observa que los grupos de [L1-] tienen mejores resultados que los grupos de $[\mathrm{L} 1+]$ en las cuatro estructuras. Según los resultados ofrecidos en la prueba de efectos intersujetos del ANOVA, el tipo de lengua ([L1+] o [L1-]) parece influir, ya que los datos arrojan resultados significativos en tres de las cuatro estructuras (verb parti $\mathrm{p}=, 053$; result $\mathrm{p}=, 000$; doble objeto $\mathrm{p}=, 001$; [NN] $\mathrm{p}=, 005)$.

Con respecto al contexto de aprendizaje, los grupos de VZLA tienen mejores resultados que los grupos de EEUU en todas las estructuras menos en el caso de la [NN]. El grupo de [L1+] VZLA tiene resultados mucho mejores que los de su par en EEUU. Hay que resaltar que en situación de aprendizaje instruccional + natural el grupo [L1+] VZLA mejora en gran medida con respecto a su par en contexto instruccional (EEUU). El contexto por sí solo también parece influir porque arroja resultados significativos en las 4 estructuras (verb parti $\mathrm{p}=, 001$; result $\mathrm{p}=, 025$; doble objeto $p=, 026$; [NN] $p=, 032$ ).

Con referencia a la interacción contexto-lengua, la prueba de efectos intersujetos del ANOVA solo arroja resultados significativos para la estructura [NN] $(p=, 014)$, las otras estructuras arrojan los siguientes resultados (verb parti $\mathrm{p}=, 308$; result $\mathrm{p}=, 847$; doble objeto $\mathrm{p}=, 798$.

El gráfico $N^{\circ} 2$ muestra el desempeño de los hablantes de [L1+] y [L1-] en el uso de las estructuras [NN] y [NdeN] con núcleo a la izquierda en la prueba de selección simple (PSS) según situación de aprendizaje, tercer objetivo de nuestro estudio. 
Gráfico 2. Comparación contexto-tipo de lengua en las dos estructuras posibles del español en el PSS

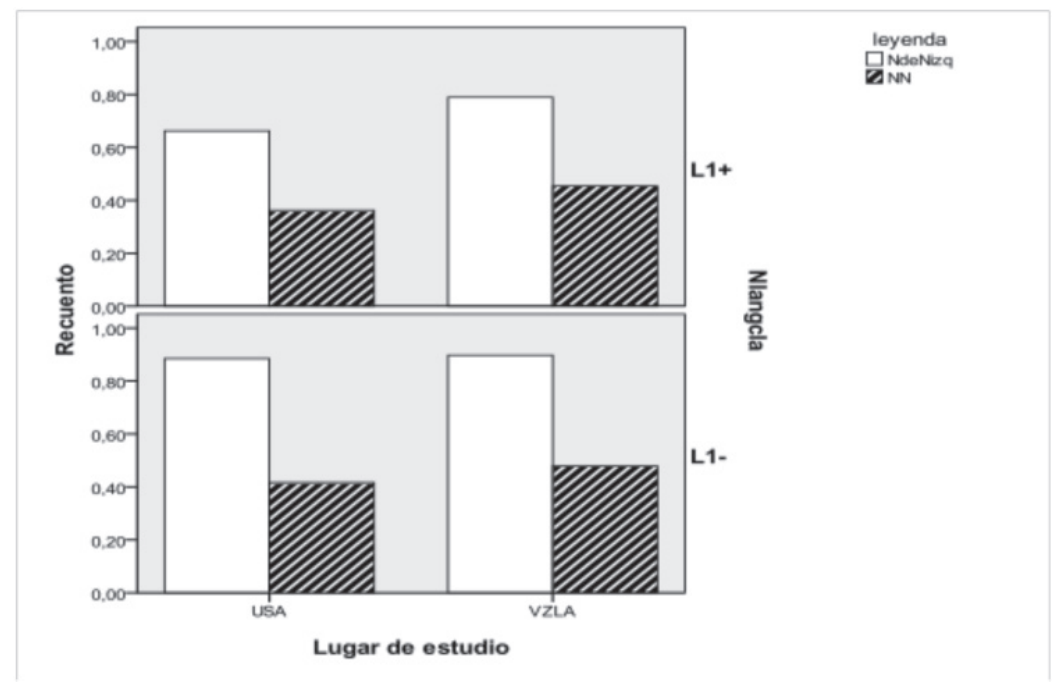

En lo que respecta al tipo de lengua, el grupo [L1-] EEUU tiene mejor desempeño ([NdeN] $=, 8846$ y [NN] ,4167) que el grupo [L1+] EEUU ([NdeN] $=, 6622$ y $[\mathrm{NN}]=$, 3625) en ambas estructuras. Por otra parte, el grupo [L1-] VZLA tiene mejor desempeño ([NdeN] $=, 8974$ y $[\mathrm{NN}]=, 4792$ ) que $[\mathrm{L} 1+]$ VZLA ([NdeN] $=, 7892$ y NN $=, 4532$ ). La prueba de efectos intersujetos del ANOVA muestra resultados significativos para la estructura [NdeN] $(p=, 001)$ pero no para la estructura $[N N](p=, 606)$. La lengua materna parece incidir en la percepción de que la estructura [NdeN] es la tendencia en español.

En cuanto al contexto, se observa que el grupo [L1-] VZLA tiene un mejor desempeño ([NdeN] $=, 8974 \mathrm{y}[\mathrm{NN}]=, 4792)$ que el de [L1-] EEUU ([NdeN] $=, 8846 \mathrm{y}[\mathrm{NN}]=, 4167)$. El contexto no parece ser $\tan$ determinante en estos grupos de [L1-] para ambas estructuras. Ahora bien, el grupo [L1+] VZLA tiene mejores resultados $([\mathrm{NdeN}]=, 7892 \mathrm{y}[\mathrm{NN}]=, 4537)$ que el grupo [L1+] EEUU ([NdeN] =,6622 y [NN] $=, 3625)$ en ambas estructuras. El contexto pareciera ser más determinante en este caso. Sin embargo, en la prueba de efectos intersujetos del ANOVA no hay resultados significativos para la influencia del contexto de aprendizaje para las dos estructuras ([NdeN] $\mathrm{p}=, 147$; [NN] $\mathrm{p}=, 320$ ).
Considerando la interacción contexto-lengua, los mejores resultados se observan en [L1-] VZLA en ambas estructuras ([NdeN] y [NN]), y los peores resultados están en [L1+] EEUU en ambas estructuras. Hay que resaltar que en [L1+] la diferencia con respecto al contexto es más marcada/acentuada que en [L1-]. Pareciera que el contexto hace diferencia en el caso de [L1+] y no tanto en [L1-] aunque los datos de la prueba de efectos intersujetos del ANOVA no arrojan resultados significativos en estas estructuras ([NdeN] $\mathrm{p}=, 235 ;$ [NN] $\mathrm{p}=, 852$ ).

El gráfico $N^{\circ} 3$ muestra el desempeño de los hablantes de [L1+] y [L1-] en las estructuras [NN] y [NdeN] con versión gramatical (núcleo a la izquierda) y agramatical (núcleo a la derecha) en la PSS según la situación de aprendizaje (objetivo $\mathrm{N}^{\circ} 4$ del estudio).

Al examinar los resultados observamos que el [NdeNizq] ${ }^{5}$ es la opción más escogida por los sujetos de estudio independientemente de su lengua y de la situación de aprendizaje. Esta estructura es también la opción correcta para la mayoría de los ítems, 13 de los 17 ítems de la PSS. Asimismo, la estructura

5 [NNizq] representa la estructura [NN] con núcleo a la izquierda (versión gramatical en español). [NdeNizq] representa la estructura [NdeN] con núcleo a la izquierda (versión gramatical en español). 
[NdeNder] y [NNder] ${ }^{6}$ constituyen las opciones menos seleccionadas por los sujetos, salvo en el caso de [L1-] EEUU que presenta una ligera preferencia por [NNder].

Gráfico 3. Estructuras gramaticales y no-gramaticales en la PSS

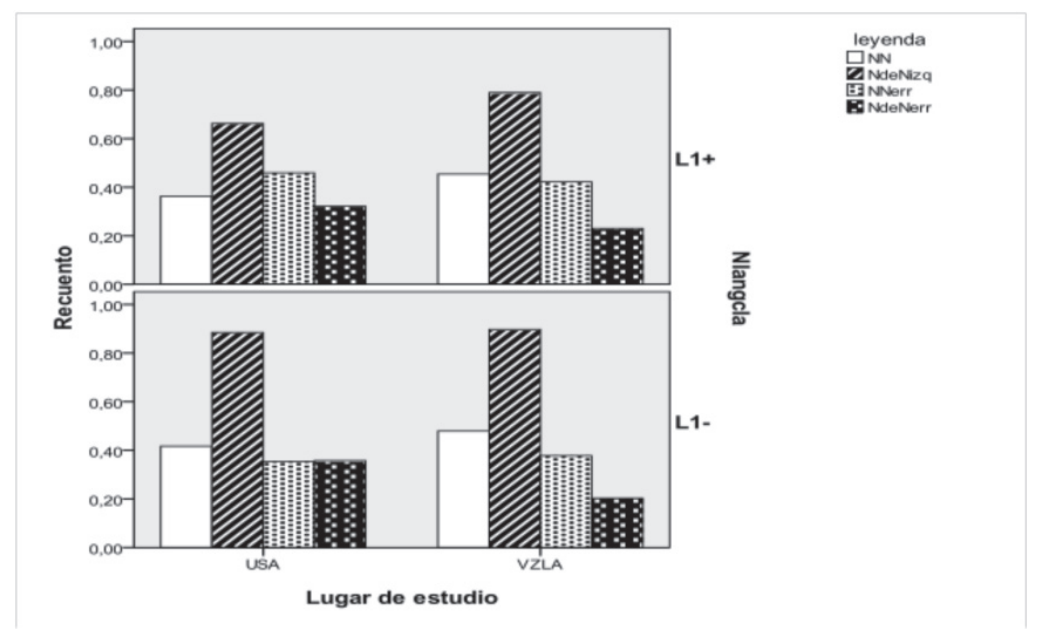

Con respecto al tipo de lengua, [L1-] escoge más [NdeNizq] (EEUU $=, 8846$ y VZLA $=, 8974$ ) que $[\mathrm{L} 1+] \quad(\mathrm{EEUU}=, 6622$ y VZLA $=, 7892)$ en ambas situaciones de aprendizaje. La prueba de efectos intersujetos del ANOVA arroja resultados significativos para esta estructura en relación con la lengua materna [NdeNizq] $p=, 001$.

[L1+] selecciona más [NNder] (EEUU $=, 4588$ y VZLA $=, 4220)$ que [L1-] (EEUU $=, 3529$ y VZLA $=, 3775$ ) independientemente de su situación de aprendizaje. La prueba de efectos intersujetos del ANOVA arroja resultados significativos para esta estructura en relación con la lengua materna [NNder] $\mathrm{p}=, 014$.

En cuanto al contexto, en lo que concierne a las estructuras de [NN] izquierda o derecha, es interesante notar que [L1+] VZLA, [L1-] EEUU y [L1-] VZLA prefieren la estructura [NNizq] a la [NNder], mientras que [L1+] EEUU selecciona mayormente la [NNder]. En lo que concierne a las estructuras [NdeN] izquierda o derecha, los sujetos de [L1+] VZLA obtienen mejores resultados para la [NdeNizq] que sus pares en EEUU (promedio $=, 7892$ versus promedio $=, 6622$ ). La estructura [NdeNder] es más seleccionada por EEUU $([\mathrm{L} 1+]=, 3214$ y $[\mathrm{L} 1-]=, 3571)$ que por VZLA ([L1+] $=, 2301$ y $[\mathrm{L} 1-]=, 2024)$ independientemente de la lengua materna. La prueba de efectos intersujetos del ANOVA resulta significativa para la estructura en relación con contexto [NdeNder] $p=, 023$, lo cual hace pensar que el contexto tiene un papel importante.

En cuanto a la interacción contexto-lengua, el estudio no ofrece resultados significativos para este aspecto.

\section{Conclusión y discusión}

Los mejores resultados obtenidos en ambas pruebas son para los hablantes de [L1-] en el contexto de aprendizaje natural+instruccional mientras que los resultados más bajos los ofrecen los hablantes de $[\mathrm{L} 1+]$ en el contexto instruccional, lo que constituye el

6 [NNder] representa la estructura [NN] con núcleo a la derecha (versión agramatical en español). [NdeNder] representa la estructura [NdeN] con núcleo a la derecha (versión agramatical en español). 
efecto esperado. En el contexto natural + instruccional el input y la lengua materna parecen tener un papel determinante.

En la PJG los resultados significativos que se muestran parecen indicar que el contexto natural+instruccional influye positivamente en la adquisición de las 4 estructuras tratadas en esta prueba. Por su parte en el caso de la lengua, los resultados significativos indican que la lengua materna parece constituir un elemento favorecedor en la adquisición de 3 de las 4 estructuras empleadas. En el caso del verbo partícula, para el cual el análisis ANOVA no muestra resultados significativos, la ausencia de esta estructura en el español y la falta de evidencia negativa podrían explicar este resultado. Al cruzar las variables contexto-lengua, solamente hay resultados significativos para la estructura [NN], el aprendiente pareciera darse cuenta casi inmediatamente de la forma como funciona el [NN] en el español.

Con respecto al PSS, el contexto parece ser más determinante en el caso de los hablantes de [L1+] que en el de los de [L1-] aunque no hay resultados significativos. La lengua materna parece incidir en la percepción de que la estructura [NdeNizq] es la tendencia en español. Asimismo, la lengua materna parece tener un papel importante en el hecho de que se dé preferencia a la estructura [NdeNizq] y no a la estructura [NNder]. Los hablantes de [L1-] parecen tener una ventaja con respecto a los de [L1+] dado que una buena parte de ellos se da cuenta de que la forma [NNder] no existe en el español y que la [NdeN] siempre tendrá el núcleo a la izquierda. Por otra parte, el contexto parece jugar un papel importante con respecto al desempeño de [NdeNder] lo que podría explicarse por la ausencia de esta estructura en el input.

Al comparar los resultados de nuestro estudio con el de Slabakova (2002) en la PJG, encontramos coincidencias en cuanto a que el desempeño general de los aprendientes de ambos estudios ofrece buenos resultados para las estructuras [NN] y doble objeto y peores resultados para verbo partícula y resultativo, tal como reporta Slabakova (2002). Según esta autora, los aprendientes parecen fijar el valor del español para una parte de los correlatos del parámetro pero no para la otra; es decir, que no parecen adquirirse como un cluster, como podría deducirse del Parámetro de los Compuestos. En el caso de la PSS también encontramos coincidencias con el estudio de Slabakova (2002) en cuanto al reconocimiento de que la estructura [NdeNizq] es la tendencia en el español y que es posible la presencia de [NN]. Sin embargo, hay que destacar que el estudio de Slabakova (2002) considera niveles de adquisición de la lengua meta, las lenguas de los sujetos experimentales son solamente anglófonas y francófonas mientras que los sujetos experimentales de nuestro estudio son hablantes nativos de muchas otras lenguas clasificadas siguiendo la tipología de Snyder (1995, 1996, 2001). Por otra parte, el instrumento empleado por Slabakova (2002) ofrece solo opciones del tipo [NN] incorrecto, mientras que nuestro instrumento ofrece opciones de [NN] correcto e incorrecto.

Al comparar los resultados de nuestro estudio con los de Liceras et al. (2001) y los de Liceras et al. (2002) observamos algunas semejanzas y varias diferencias. En estos dos estudios la estructura [NN] con núcleo a la izquierda o a la derecha constituye una estrategia muy productiva. Por el contrario, los sujetos de nuestro estudio tienen una preferencia marcada por la estructura [NdeNizq] en ambos grupos experimentales independientemente de la lengua y del contexto. Nuestros sujetos escogen como segunda opción la estructura [NNizq] excepto para el grupo [L1+] de EEUU quienes se comportan de manera similar a los sujetos principiantes de Canadá (en contexto instruccional) del estudio de Liceras et al. (2001) y a los sujetos finlandeses (en contexto instruccional) del estudio de Liceras et al. (2002), los cuales tienen preferencia por la estructura [NNder]. La direccionalidad de la estructura [NdeN] no es un problema mayor para los sujetos del contexto natural+instruccional de nuestro estudio, como sí lo 
es para nuestros sujetos del contexto instruccional y los de Liceras et al. (2001) y los sujetos finlandeses del Liceras et al. (2002).

En el PJG los sujetos de nuestro estudio reconocen que la estructura [NN], la cual es errónea en la prueba porque son todas con núcleo a la derecha, no es una estrategia productiva para el español y ofrecen buenos resultados al juzgar la agramaticalidad de la misma. En la PSS los sujetos seleccionan en su mayoría la estructura [NdeN] con núcleo a la izquierda y luego, en un porcentaje ya no tan elevado, la estructura [NN] con núcleo a la izquierda, salvo en el caso de los sujetos de [L1+] EEUU. Al comparar estos resultados con los ofrecidos en Trías et al. (2011), se observan algunas coincidencias ya que en ambos estudios la mayoría de los sujetos prefieren estructuras con núcleo a la izquierda tanto que sea [NdeN] como [NN], los sujetos parecen darse cuenta de que la forma [NdeN] con núcleo a la izquierda es la forma más común del español. Sin embargo, en el presente estudio el grupo de [L1+] EEUU, aunque prefiere la estructura [NdeNizq], también selecciona un número importante de [NNder]. Es importante notar que en el estudio de Trías et al. (2011) el instrumento empleado fue una prueba de evocación mientras que en el presente estudio se usaron dos pruebas (PJG y PSS).

\section{Referencias}

Bley-Vroman, R. (1989). What is the logical problem of foreign language learning? en S. Gass \& J. Schachter, (eds.) Linguistic perspectives on second language acquisition: 41-68. Cambridge: Cambridge University Press.

Comrie, B. (ed.) (1990). The World's Major Languages. New York, N.Y. Oxford University Press.

Demonte, V. (1995). "Dative alternation in Spanish", en Probus, 7: 5-30.

Gass, S. M. (1997). Input, interaction, and the second language learner. Mahwah, NJ: Lawrence Erlbaum Associates, Publishers.

Gass, S. M. (2003). Input and Interaction, en C. J. Doughty \& M. H. Long (eds.) The Handbook of Second Language Acquisition. Blackwell Publishing Ltd.: 224-255.
Gass, S. M. y Selinker, L. (2008). Second Language Acquisition. An introductory course. New York. Taylor \& Francis. (Tercera edición).

Isobe, M. (2007). The Acquisition of Nominal Compounding in Japanese: A Parametric Approach. Proceedings of the 2nd Conference on Generative Approaches to Language Acquisition North America (GALANA) (ed.) Alyona Belikova et al. 171-179. Somerville, MA: Cascadilla Proceedings Project. Disponible en http://www.lingref.com/cpp/galana/2/ paper1558.pdf

Liceras, J. y Díaz, L. (2001). "Triggers in L2 Acquisition: The Case of Spanish N-N Compounds", en Studia Lingüística, 54(2):197-211. Disponible en http:// www.ucm.es/info/circulo/no5/licerasdiaz.htm

Liceras, J., Salomaa-Robertson, T. y Díaz, L. (2002). El papel del parámetro de los compuestos y de la hipótesis del marcador palabra en la adquisición de compuestos nominales del español como segunda lengua, en A. T. Pérez-Leroux y J.M. Liceras (eds.) The Acquisition of Spanish Morphosyntax (The L1/ L2 Connection): 209-237. Dordrecht: Kluwer Academic Publishers.

Slabakova, R. (2002). "The Compounding Parameter in Second Language Acquisition", en Studies in Second Language Acquisition, 9: 157-173. Disponible en http://www.uiowa.edu/ linguist/faculty/ slabakova/personal/SSLA.pdf

Snyder, W. (1995). Language Acquisition and Language Variation: The Role of Morphology. Tesis de Doctorado. Cambridge, Massachusetts Institute of Technology. Inédita.

Snyder, W. (1996). The Acquisitional Role of the SyntaxMorphology Interface: Morphological Compounds and Syntactic Complex Predicates. Proceedings of the $20^{\text {th }}$ Annual Boston University Conference on Language Development, 2: 728-735. Somerville, MA: Cascadilla Press. Disponible en http://web2.uconn. edu/snyder/papers/WS BU95.pdf

Snyder, W. (2001). "On the Nature of Syntactic Variation: Evidence from Complex Predicates and Complex Word-formation", en Language, 77: 324-343.

Snyder, W. (2005). Motion Predicates and the Compounding Parameter: A New Approach. Disponible en http://web2.uconn.edu/snyder/papers/Maryland05. pdf

Snyder, W. y Lillo-Martin, D. (2005). Motion Predicates and the Compounding Parameter. Abstract of talk in Nanzan Linguistics $N^{\circ}$ 2. Research Results and Activities 2004, 103-5. Nagoya, Japan: Center for Linguistics, Nanzan University. Disponible en http:// web2.uconn.edu/snyder/papers/Nanzan05.pdf 
Suguisaki, K. e lsobe, M. (2000). Resultatives Result from the Compounding Parameter: On the Acquisitional Correlation between Resultatives and N-N Compounds in Japanese. WCCFL 19 Proceedings, Billerey and Lillehaugen (ed.), 493-506. Somerville, MA: Cascadilla Press. Disponible en http://faculty.human. mie-u.ac.jp/ sugisaki/Sugisaki\%26lsobe_00.PDF

Suguisaki, K. y Snyder, W. (2002). Preposition Stranding and Compounding Parameter: Developmental Perspective. Disponible en http://faculty.human.mie-u. ac.jp/ sugisaki/Sugisaki\%26Snyder 02.pdf

Talmy, L. (1985). Lexicalization patterns: Semantic structure in lexical forms, en T. Shopen (ed.) Language Typology and Syntactic Description, Volume III: Grammatical Categories and the Lexicon: 57-149. Cambridge, U.K. CUP. Disponible en http://dingo. sbs.arizona.edu/ hharley/courses/PDF/TalmyLexicalizationPatterns.pdf

Talmy, L. (1991). "Path to realization: a typology of event conflation", en Berkeley Linguistic Society, 7: 480-519.
Talmy, L. (2000). Toward a Cognitive Semantics. (Vols. 1 y 2) Cambridge, MA, MIT Press.

Trías, M. y Villanueva, AM. (2011). "La composición nominal en la en la adquisición del español como segunda lengua. Respuestas a una prueba de evocación", en Entre Lenguas, 16: 63-78.

Varela, S. (2005). Morfología léxica: la formación de palabras. Madrid. Ed. Gredos.

White, L. (1989). Universal Grammar and Second Language Acquisition. Amsterdam. John Benjamins Publishing Co.

White, L. (2000). Second language acquisition: From initial to final state, en J. Archibald (ed.), Second language acquisition and linguistic theory. Malden, MA: Blackwell Publishers Ltd: 130-155.

White, L. (2003). Second Language Acquisition and Universal Grammar. Cambridge University Press.

\section{AUTORES}

ANA MARÍA VILLANUEVA, Lic. en Idiomas Modernos de la Universidad Central de Venezuela y Magíster en Lingüística Aplicada de la Universidad Simón Bolívar. Profesor de la misma Universidad. Desde el año 2007 está encargada de la sub-sección de Francés con Fines Comunicativos Generales para los estudiantes regulares de la USB y en la enseñanza del Español como Lengua Extranjera (ELE) para los estudiantes extranjeros del Programa de Intercambio.

MARTA TRÍAS Magister en enseñanza del inglés como lengua extranjera de la Universidad Central de Venezuela. Actualmente trabaja en el departamento de lenguas de Universidad Simon Bolivar donde enseña inglés y español como lengua extranjera. 